\title{
Case Report: Coronary Dissection after High-Energy Blunt Chest Trauma
}

\author{
Garcia I, Gómez A, Paula Carmona*, Zarragoikoetxea I and Argente P \\ Anesthesia and Critical Care Hospital La Fe, Spain
}

\section{Introduction}

Coronary artery dissection in a blunt chest trauma is a rare injury and its clinical diagnosis is difficult due to symptoms related to the multiple trauma. There is lack of scientific evidences regarding the management of this pathology.

\section{Case Report}

We present the case of a 44-year-old male, without a relevant medical history, admitted to the ICU after a multiple trauma caused by a high-energy traffic accident. The patient was initially conscious and oriented with Glasgow 14 and without neurological symptoms.

After admission, a complete body CT was performed where a small non-displaced lineal fracture in the sternum, minimal pneumomediastinum and mediastinic fat edema was observed without any intracranial lesions.

Blood test after admission showed increased levels of high-sensitive troponine. In EKG tracing a $2 \mathrm{~mm}$ ST elevation in the anterior leads (V2-V4) was detected. It was related to a likely myocardial contusion. An expectant approach was therefore decided.

The second high-sensitive troponin determination reached maximum values of $3900 \mathrm{ng} / \mathrm{ml}$, and a transthoracic echocardiogram was performed, where hypokinesia in the mid and apical segments of the left ventricle's anterior wall and good global left ventricular systolic function was identified (Video 1). Due to high suspicion of coronary injury, a coronary CT was requested and a dissection of the anterior descending coronary artery was identified. Due to haemodynamic stability and haemorrhage risk after trauma no further treatments were administered.

24 hours later the patient suffered sudden neurological deterioration and was intubated. We conducted a brain CT that revealed an intraparenchymal haemorrhage. The patient remained in the ICU for 12 days with complete neurological recovery. Another coronary CT was carried out with no changes compared to the previous. After satisfactory evolution, the patient was discharged from our unit.

\section{Discussion}

Blunt cardiac trauma (BCT) usually occurs after high ener- gy collisions. Approximately, $80 \%$ of BCT are associated with significant damage to systemic organs and coronary artery dissection (Figure 1) is one of them [1].

Coronary dissection is an infrequent lesion, described by Kohli, et al. [2] in 1988. Its incidence is approximately $10 \%$ of blunt cardiac trauma cases [3]. Direct contusion on heart's anterior wall and aortic deformation during trauma are the most frequent physical mechanisms. In general, the anterior descending coronary artery is the most affected, followed

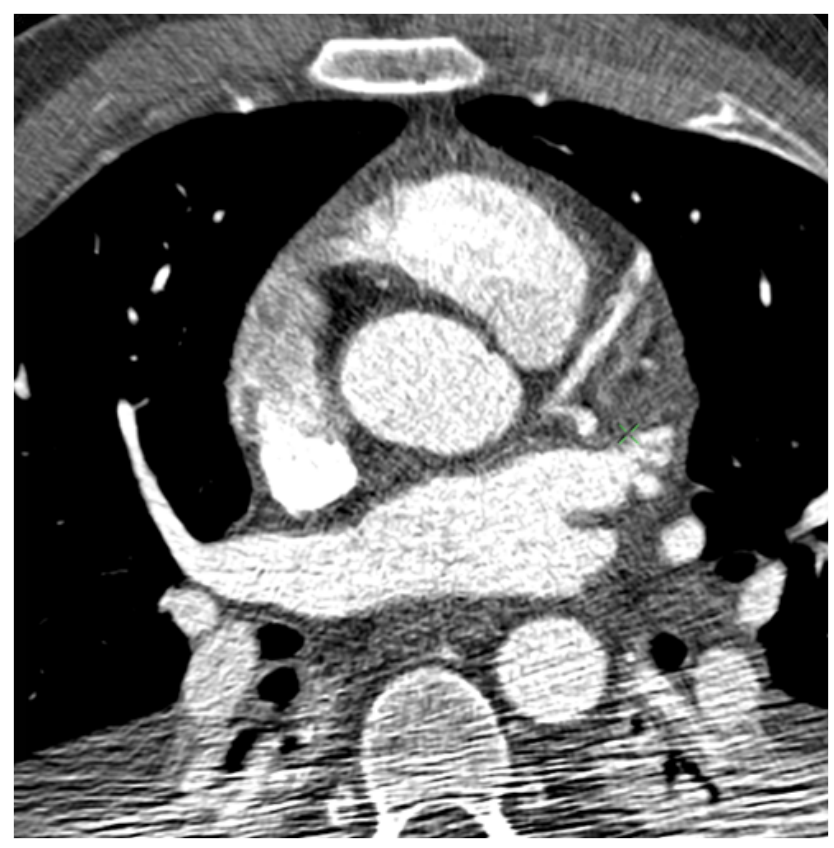

Figure 1: Artery dissection coronary TC.

*Corresponding author: Paula Carmona, Anesthesia and Critical Care Hospital La Fe, Valencia, Spain

Accepted: June 10, 2019

Published online: June 12, 2019

Citation: Garcia I, Gómez A, Carmona P, et al. (2019) Case Report: Coronary Dissection after High-Energy Blunt Chest Trauma. J Cardiothorac Surg Ther 3(1):31-32 


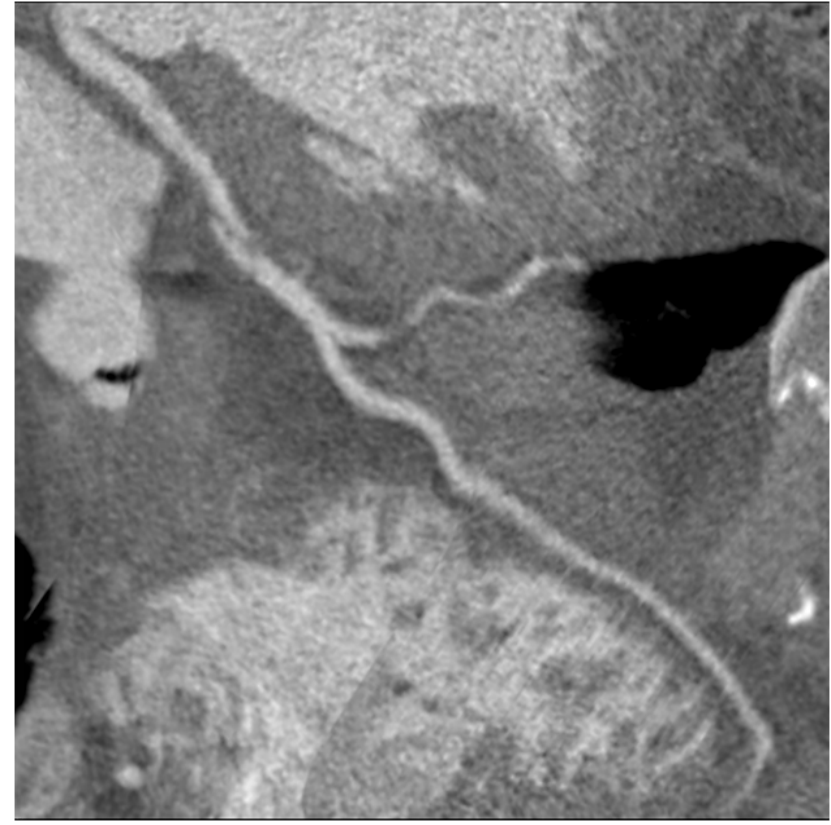

Figure 2: 3D TAC reconstruction.

by the right coronary artery. Arteriosclerosis seems to be a predisposing factor [4].

Thoracic pain is a common and unspecific symptom of the multiple trauma patient and the diagnosis of coronary pathology requires a high level of clinical-suspicion. Therefore complementary explorations are necessary for the diagnosis. The elevation of myocardial enzymes and changes in the EKG are the main signs in a patient with BCT [5]. Echocardiography is critical in the diagnosis, therapeutic decisions and monitoring. The evidence of segmental wall motion contractility abnormalities supports the diagnose of coronary lesion. Global systolic ventricular function assessment is mandatory to optimize the haemodynamic management of these patients and helps in the indication of a more invasive treatment, such as coronary angiography, coronary stenting or even coronary artery bypass grafting.

Once echocardiographic findings suggest a coronary artery dissection, another technique to confirm the diagnosis is required. The coronary CT is a very useful test without the risks associated to a more invasive procedures such as coronary angiography which requires heparinization (Figure 2).

Treatment of spontaneous coronary dissections due to lack of scientific evidence and associated haemorrhage risk is controversial. Medical treatment with antiplatelet is not generally indicated. In case of indication acetylsalicylic acid is the most recommended. The association with clopidogrel, prasugrel o ticagrelor has not been widely studied and Gpllb/ Illa inhibitors are not used due to their higher risk of bleeding [6].

Other more invasive treatments, such as percutaneous stenting or CABG might be considered in hemodynamic unstable patients with global systolic dysfunction. The indication of medical or invasive treatment in multiple trauma patient is determined by the risk of haemorrhage particularly in the case of brain trauma which might have devastating consequences.

\section{Conclusions}

Diagnosis and treatment of coronary dissection in the context of a multiple trauma patient is complicated and lacks of sufficient scientific evidence. EKG changes together with enzymatic increases should suggest the diagnosis. Echocardiography is a key tool in the management of this pathology guiding the diagnosis through the demonstration of wall motion abnormalities. Coronary CT shows the anatomy of the injury without the risks associated to heparin administration. Definitive treatment will depend on the clinical status, echocardiographic findings and associated trauma lesions.

\section{References}

1. Raid Yousef, John Alfred Carr (2014) Blunt cardiac trauma: A Review of the current knowledge and management. Ann Thorac Surg 98: 1134-1140.

2. Kohli S, Saperia GM, Waksmonski CA, et al. (1988) Coronary artery dissection secondary to blunt chest trauma. Cathet Cardiovasc Diagn 15: 179-183.

3. Brasseur E, Ghuysen A, Mommens V, et al. (2006) Coronary dissection and non-penetrating chest trauma. Ann Cardiol Angeiol 55: 233-239.

4. Federico Colombo, Andrea Zuffi, Alessandro Lupi (2014) Left main dissection complicating blunt chest trauma: Case report and review off literature. Cardiovasc Revasc Med 15: 354-356.

5. Magdalena Wilczynska-Golonka, Rostoff P, Siniarski A, et al. (2017) Trauma-induced acute myocardial infarction due to delayed dissection of the left anterior descending coronary artery. Am J Emerg Med 35: 939.

6. Amelia Yip, Jacqueline Saw (2015) Spontaneous coronary artery dissection-A review. Cardiovasc Diagn Ther 5: 37-48.

DOI: $10.36959 / 582 / 416$

Copyright: (c) 2019 Garcia I, et al. This is an open-access article distributed under the terms of the Creative Commons Attribution License, which permits unrestricted use, distribution, and reproduction in any medium, provided the original author and source are credited. 